\title{
Preventative Effect of Sugars on the Pollen Degeneration of Wheat Plant*
}

\author{
by Hirosuke FukasawA**, Katsuhiro Mito** and Masanobu Fujiwara**
}

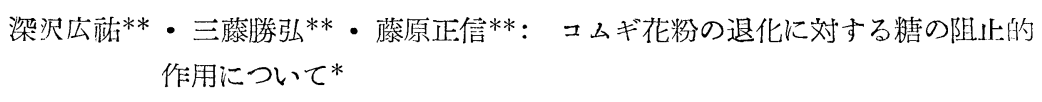

Received April 8, 1957

\section{Introduction}

Emmer wheat with the cytoplasm of Aegilops ovata obtained by means of successive backcrosses is characterized by its male-sterility (Fukasawa, 1953). Cytological investigation on the pollen degeneration in the sterile anthers, shows that microspore development proceeds normally with complete pairing of 14 bivalents at meiosis. After liberation from tetrads the microspores fail to develop, degenerating in the course of pollen mitosis (Fukasawa, 1956).

A similar degeneration of pollen grains can be induced artificially in normal wheat plants. For example, when stalked young spikes with two leaves are cut off at the stage of pollen mitosis and placed in the dark for about one week, white spikes without chlorophyll emerge from the leaf-sheath, and no good pollen grains are formed (Fukasawa, 1956). The degree of pollen degeneration in the dark increases extremely when the plant is cut off at the meiotic stage. The present report deals with the experimental results concerning the restoration of pollen formation by supplying sugar to the culture solution in the dark, and a preliminary attempt to resolve the mechanism of pollen degeneration in cytoplasmic male-sterile wheat. In addition the study deals with an attempt to prevent the degeneration of pollen grains in the male-sterile wheat, and a paper chromatographic analysis to survey the sugars present in male-sterile and normal anthers.

\section{Materials and Methods}

The wheat plants used in the present investigation are Triticum durum var. Reichenbachii and two male-sterile strains with the cytoplasm of Ae. ovata, namely, male-sterile durum and male-sterile dicoccum (Khapli). These plants were planted in the field. Stalked young spikes enveloped in the leaf-sheath were, with two leaves, cut off at the meiotic stage, and placed in a bottle with Shive's solution.

\footnotetext{
* Contributions from the Biological Institute, Faculty of Science, Kobe University, No. 43.

** Biological Institute, Facutly of Science, Kobe University, Kobe, Japan 神戸大学理学部生物学 教窒
} 
Normal spikes of $T$. durum were cultured in the dark at room temperature for about one week or more. Sugars added in the culture solution were glucose, fructose or sucrose in a concentration of $5 \%$ or $7 \%$. Male-sterile spikes were placed in daylight at room temperature, adding such sugars to Shive's solution or tap water as $0.5 \%, 1 \%, 3 \%$ and $5 \%$ sucrose, $3 \%$ glucose or $0.5 \%$ and $1 \%$ fructose.

Pollen grains were investigated by staining with dilute aceto-carmine solution to detect any abnormality in nuclear division and cytoplasmic anomalies in the course of sporogenesis. Various types of pollen degeneration were classed in the following five grades according to Kihara (1937). Namely: class-I, normal good pollen with one vegetative nucleus and two generative nuclei; class-II, pollen grains with three not fully differentiated nuclei; class-III, two nucleate pollen; class-IV, one nucleate pollen; class- $\mathrm{V}$, empty pollen.

The survey of sugars in the anthers was made by means of ascending one-dimensional paper-chromatography. Sample solutions were prepared by extracting the anthers with $50 \%$ ethy1 alcohol. Development of sugars was carried out by the use of a mixture of $n$-butanol, acetic acid and water (5:1:2). After the filter paper was dried at room temperature, it was sprayed with alcoholic benzidine solution (benzidine 0.5 g., acetic acid 10 cc., absolute alcohol 80 cc., and $40 \%$ trichloroacetic acid $10 \mathrm{cc}$.) and heated until the clear brown spots appeared.

\section{Experimental Results}

1) Normal plant of $T$. durum in the dark.

When the stalked spikes of normal plant of T. durum were placed in the dark, they grew very slowly and, the green color of their leaves and leaf-sheaths faded gradually. White and tender spikes emerged after one week or more. However, when $5 \%$ glucose, sucrose or fructose was added to the culture medium, they grew more rapidly than the control without sugar (Fig. 1); the decoloration of the leaves and sheaths was also less conspicuous than with the control plants, and the heads were composed of rather tough spikes with a light yellow color.

Results of investigation of pollen grains obtained from these spikes are presented in Table 1. Normal plants growing in the field were found to produce as much as $95 \%$ good pollen grains, whereas on the plants cultured in the dark, most of microspores became either one-nucleate (class-IV) or empty (class-V). Good pollen (class-I) was not produced at all. However, when sugar (glucose or sucrose) was added to the culture solution, a considerable amount of good pollen grains was produced (Table 1). For example, the spikes grown in $5 \%$ sucrose solution produced as many good pollen grains $(92 \%)$ as the normal spikes growing in the field. When $7 \%$ glucose was used, $59 \%$ of the microspores grew into good pollen grains of class-I, $26 \%$ were class II, the rest of them belonged to the class-III, -IV, and $-\mathrm{V}$. In order to confirm the results obtained in the above experiment (exp. 1 , 


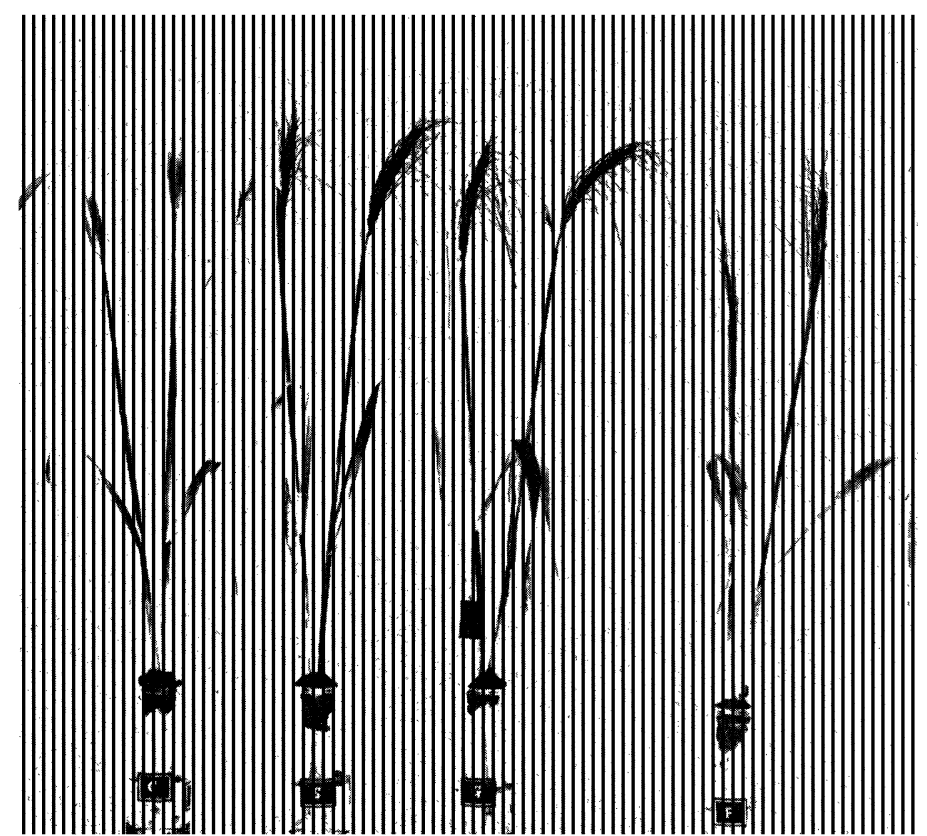

Fig. 1. Dark-grown spikes of Triticum durum. C: control (no sugar), S: sucrose, G: glucose, F : fructose

Table 1. Pollen analysis in the dark-grown spikes of Triticum durum

\begin{tabular}{|c|c|c|c|c|c|c|}
\hline \multirow{2}{*}{ Experiment no. } & \multirow{2}{*}{ Culture conditions } & \multicolumn{5}{|c|}{ Pollen classes } \\
\hline & & I & II & III & IV & $\mathrm{V}$ \\
\hline \multirow{5}{*}{$\begin{array}{c}1 \\
(\text { May } 9,1956)\end{array}$} & In field & $95.4 \%$ & $0.4 \%$ & $2.7 \%$ & $0.9 \%$ & $0.6 \%$ \\
\hline & $\begin{array}{l}\text { Tap water in the } \\
\text { light }\end{array}$ & 92.4 & 0.6 & 3.2 & 2.2 & 1.6 \\
\hline & Control (no sugar) & 0 & 0.5 & 12.4 & 47.1 & 40.0 \\
\hline & $5 \%$ sucrose & 92.1 & 4.4 & 2.8 & 0.2 & 0.5 \\
\hline & $7 \%$ glucose & 58.6 & 25.6 & 8.7 & 3.4 & 3.7 \\
\hline \multirow{4}{*}{$\begin{array}{c}2 \\
\text { (June } 4,1956 \text { ) }\end{array}$} & $5 \%$ sucrose & 37.3 & 44.2 & 13.1 & 4.4 & 1.0 \\
\hline & $7 \% \quad 11$ & 18.1 & 43.3 & 32.2 & 4.4 & 2.0 \\
\hline & $5 \%$ glucose & 0 & 11.8 & 78.4 & 9.3 & 0.5 \\
\hline & $7 \% \quad \prime \prime$ & 0.2 & 14.4 & 78.4 & 6.8 & 0.2 \\
\hline 3 & $5 \%$ sucrose & 44.4 & 37.1 & 13.8 & 3.9 & 0.8 \\
\hline (June 15,1956 ) & $5 \%$ glucose & 2.6 & 35.7 & 56.6 & 3.1 & 2.0 \\
\hline
\end{tabular}

Table 1), two sets of experiments were performed in June, with the intention of deciding whether sucrose or glucose was more effective in ameliorating the development of microspore. The results (exp. 2 and 3, Table 1) showed the same trend of restoration of normal pollen production although the yield of good pollen (classI) was not always as marked as in the earlier experiment. The low fertilities in 
the 2nd the 3rd experiments may be due to the unappropriate condition of the test plants investigated here in rather late season. Five percent sucrose was, however, found more suitable for the production of good pollen grains than $7 \%$ sucrose, $5 \%$ glucose or $7 \%$ glucose.

2) Male-sterile durum plant in the daylight.

When the stalked young spikes of male-sterile durum plant were cultured in the daylight, as seen in Fig. 2, the spikes in sucrose solution grew better than those in the medium without sugar, having almost the same appearance as in a normal spike of T.durum. Pollen analysis was made with such a male-sterile spikes. Pollen grains from male-sterile durum plants growing in the field showed a higher degree of degeneration in this season than in the previous year (cf. Table 1, Fukasawa, 1956), i.e., a large number of pollen was empty and no good pollen of class I and II was observed (there were about $2 \%$ class-II pollen last year). The addition of sugar to the medium was found to be efficacious to some extent in improving the pollen status of those male-sterile wheat plants, although no first class pollen (class-I) was actually produced by the treatment. The results are summarized in Table 2. The addition of fructose, glucose or sucrose to the culture solution decreased the percentage of empty pollen grains to about $30-40 \%$ as comparaed to $70 \%$ in the control without

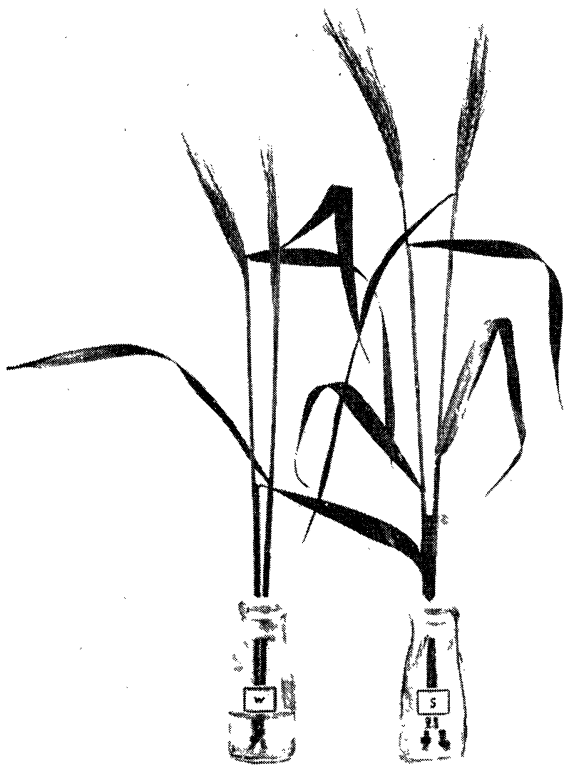

Fig. 2. Male-sterile durum plant cultured in the daylight. W: control (tap water, no sugar), S: $3 \%$ sucrose. sugar. The yield of class-IV pollen was increased to about $50 \%$ as compared to $25 \%$ in the control. The percentage of class-III pollen was also increased by the artificial supply of sugars, especially sucrose. Somewhat better results were obtained with sucrose; in fact, the production of class-II pollen was narrowly realized. The ameliorative effect of glucose was about the same with male-sterile dicoccum plants.

3) Paper chromatography of sugars in the anthers.

From the above experimental results it was found that the addition of sugars to the culture medium prevents the pollen degeneration in normal wheat plants placed in the dark, and promotes pollen development in male-sterile anthers. Since sucrose seemed to be most favorable in promoting the normal pollen production, chromato- 
Table 2. Pollen analysis in male-sterile spikes grown in the daylight, adding sugars

\begin{tabular}{|c|c|c|c|c|c|c|}
\hline \multirow{2}{*}{ Strains } & \multirow{2}{*}{ Culture conditions } & \multicolumn{5}{|c|}{ Pollen classes } \\
\hline & & I & II & III & IV & $\mathrm{V}$ \\
\hline \multirow[t]{8}{*}{ Male-sterile durum } & In field & $0 \%$ & $0 \%$ & $4.4 \%$ & $25.7 \%$ & $69.9 \%$ \\
\hline & $0.5 \%$ fructose & 0 & 0 & 9.9 & 56.0 & 34.1 \\
\hline & $1.0 \% \quad \prime \prime$ & 0 & 0 & 2.1 & 57.7 & 40.2 \\
\hline & $3.0 \%$ glucose & 0 & 0 & 9.6 & 62.0 & 28.4 \\
\hline & $0.5 \%$ sucrose & 0 & 1.7 & 15.3 & 55.6 & 27.4 \\
\hline & $1.0 \% \quad " \prime$ & 0 & 3.0 & 17.2 & 52.9 & 26.9 \\
\hline & $3.0 \%$ & 0 & 0 & 2.1 & 38.1 & 59.8 \\
\hline & $5.0 \% \quad \prime \prime$ & 0 & 0.5 & 17.6 & 53.8 & 28.1 \\
\hline \multirow{4}{*}{$\begin{array}{l}\text { Male-sterile } \\
\text { dicoccum (Khapli) }\end{array}$} & In field & 0 & 0.1 & 21.7 & 32.4 & 45.8 \\
\hline & $0.5 \%$ glucose & 0 & 3.6 & 20.1 & 54.2 & 22.1 \\
\hline & $1.0 \% \quad " \prime$ & 0 & 1.4 & 20.4 & 49.3 & 28.9 \\
\hline & Control (no sugar) & 0 & 0 & 1.3 & 3.3 & 95.4 \\
\hline
\end{tabular}

graphic analysis of sugars in male-sterile and normal anthers was made with the purpose of detecting the possible differences in the sugar contents in normal and male-sterile anthers. Each sample of male-sterile durum and normal plants consisted of 200 anthers whose fresh weight was $45 \mathrm{mg}$. and $110 \mathrm{mg}$., respectively. Furthermore, maize anthers taken from cytoplasmic male-sterile and normal plants were tested by the same method as used for wheat anthers. Each maize sample consisted of $120 \mathrm{mg}$. material (fresh weight), which was 127 anthers in male-sterile

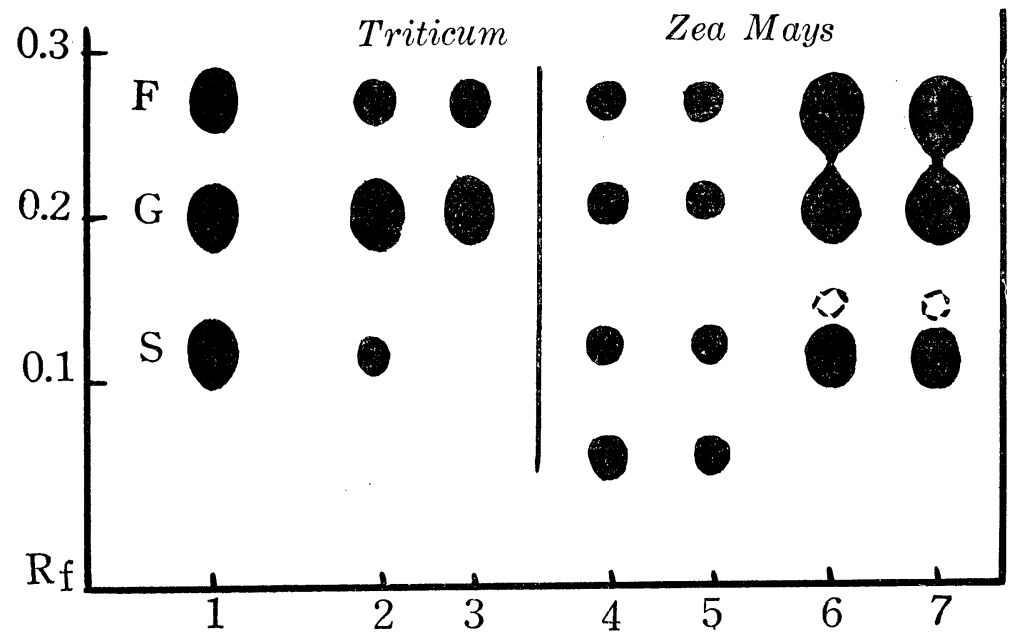

Fig. 3. Paper chromatograms of the sugars in male-sterile and normal anthers. 1 ; standard (F: fructose, G: glucose, S: sucrose), 2; normal wheat, 3; malesterile wheat, 4; young anther of normal maize, 5; young anther of male-sterile maize, 6 ; mature anther of normal maize, 7; mature anther of male-sterile maize. 
plants, and 42 in normal plants. After developing for $15 \mathrm{hrs}$. sugars were detected by spraying with benzidine solution. The results are presented in Fig. 3. Normal anthers of $T$. durum gave three spots, in which one small spot represented sucrose and two large spots fructose and glucose, respectively. Only two spots appeared in male-sterile durum anthers with no spot in the sucrose position. On the other hand, maize anthers gave spots for all three sugars, fructose, glucose, and sucrose, and both in male-sterile and normal anthers. Furthermore, young maize anthers produced four spots, of which three coincided with these described just above; another one appeared to be maltose.

\section{Discussion}

There are many reports about favorable effects of sucrose on the growth of vegetative organs in plant (Juhren and Went, 1949; Lee, 1950; Street and Lowe, 1950; Street and McGregor, 1952; Hildebrandt and Riker, 1953; Böhning, Kendall and Linck, 1953; Rietsema, Satina and Blakeslee, 1953; Frank and Kenny, 1955). However, the effect of sucrose on the development of generative organ has hardly been investigated. Iwanami (1954) reported that starch grains in the pollen of Impatiens increased in the medium with $5 \%$ sucrose. In the present investigation, it was revealed that the pollen degeneration with wheat spikes placed in the dark was prevented by the addition of sugars to the culture medium. This leads us to the supposition that sugars are indispensable for the normal formation of pollen grains. Furthermore, since sucrose was more effective than the other sugars and since paper chromatographic analysis showed no sucrose spot in the male-sterile wheat anthers, it seems likely that the presence of sucrose is essential for normal development of the pollen grains. However, adding sucrose to the young spikes of the male-sterile durum plant did not result in the formation of good pollen grains, though some progress of pollen development was found. From these facts, it may be assumed that the pollen degeneration in cytoplasmic male-sterile wheats is caused not solely by the deficiency of sucrose in the anthers but also of other still unknown factors.

As the results of his paper chromatographic study, Fukasawa (1954) has reported the disappearance of proline and the remarkable accumulation of asparagine in the anthers of cytoplasmic male-sterile wheat and maize in the course of pollen degeneration. A similar relationship between proline and asparagine was also found in the flag-leaf of male-sterile durum plant, as well as in both anthers and flag-leaf in the normal plant of $T$. durum placed in the dark for one week or more (Fukasawa and Mito, 1956). Further investigation should be carried on to determine whether such a relationship between proline and asparagine in the male-sterile plant is directly connected with the sucrose deficiency described in the present study. 


\section{Summary}

1) The present report deals with the preliminary investigations of the influence of sugar supply upon the pollen formation in wheat, in order to find a method of preventing pollen degeneration in cytoplasmic male-sterile anthers.

2) When young stalked spikes of normal wheat plant ( $\boldsymbol{T}$. durum) were cut off at the meiotic stage and placed in the dark, all microspores became abortive. Adding of sugars, especially $5 \%$ sucrose, to the culture medium protected the pollen grains from degeneration, yielding good pollen almost to the same extent as in the normal plant growing in the field.

3) When a male-sterile durum plant (stalked spikes) was cultured in the day. light, adding of sucrose did not result in the production of good pollen grains, but some progressive features of pollen development were observed in cytological investigation.

4) Paper chromatography was used for the survey of sugars in male-sterile and normal wheat anthers. Normal anthers showed three sopts: fructose, glucose and sucrose; while male-sterile anthers did not show the sucrose spot.

5) From these result, it was concluded that the lack of sucrose in the plant was one of the main causes of pollen degeneration in the cytoplasmic male-sterile wheat, althought other still unknown factors might also be involved.

\section{Literature}

1) Böhning, R. H., Kendall, W.A. and Linck, A. J., Amer. Jour. Bot. $40: 150$ (1953). 2) Frank, S. and Kenney, A.L., Plant Physiol. 30 : 413 (1955). 3) Fukasawa, H., Cytologia 18: 167 (1953). 4) __ Jap. Jour. Genet. $29: 135$ (1954). 5) ___, Cytologia $21: 97$ (1956). 6) —, and Mito, K., Kagaku 26: 313 (1956). 7) Hildebrandt, A. C. and Riker, A. J., Amer. Jour. Bot. 40 : 66 (1953). 8) Iwanami, Y., Bot. Mag. Tokyo 6ï: 135 (1954). 9) Juhren, M. C. and Went, F. W., Amer. Jour. Bot. $36: 552$ (1949). 10) Kihara, H., Mem. Coll. Agr., Kyoto Imp. Univ. $41: 1$ (1937). 11) Lee, A. E., Amer. Jour. Bot. 3i: 528 (1950). 12) Rietsema, J., Satina, S., and Blakeslee, A. F., ibid. $40: 538$ (1953). 13) Street, H. E. and Lowe, J. S., Ann. Bot. N. S. $14: 307$ (1950). 14) Street, H. E. and McGregor, S. M., ibid. $16: 185$ (1952). 